\title{
Abordaje terapéutico de la obstrucción maligna de colon. Actualización en las indicaciones de endoprótesis
}

Elena SANCHIZ CARDENAS 
Indice

1. Introducción..............................................

2. Obstrucción maligna de colon...............................3

2.1 Tratamiento quirúrgico...............................4

2.1.1. Colostomia derivativa........................5

2.1.2. Intervención de Hartmann.....................5

2.1.3. Resección primaria y anastomosis...........6

2.1.4. Colectomia total.............................6

2.1.5. Endorprótesis autoexpandible..............6

3. Endoprótesis en la obstrucción maligna de colon............7

3.1. Indicaciones....................................... 8

3.2. Contraindicaciones.................................10

3.3. Complicaciones.....................................11

3.4. Tipos y composición de las endoprótesis...............12

3.5. Preparación y procedimiento $\ldots \ldots \ldots \ldots \ldots \ldots \ldots \ldots \ldots \ldots$

4. Bibliografía........................................... 14 


\section{Introducción}

A pesar de los esfuerzos para el diagnóstico precoz del cáncer de colon con los programas de screening, más del $33 \%$ de los paciente con carcinoma colorectal debutan con síntomas que van a requerir de una intervención quirúrgica urgente tanto por perforación, obstrucción o hemorragia.

Las tasas de morbilidad, mortalidad y la necesidad e estoma son mayores en los casos con intervención urgente en comparación con la cirugía electiva. Todo esto influye no sólo en la cirugía urgente, sino en las condiciones de los pacientes intervenidos, frecuentemente en contexto de deshidratación, desnutrición, descompensación hidroelectrolitica y de comorbilidades asociadas, con tumores con un comportamiento biológico más agresivo y mayor frecuencia de metástasis hepáticas. Además, el estudio de extensión es incompleto en estas situaciones, pudiendo condicionar el abordaje oncológico posterior. Teniendo en cuenta que deben respetarse los principios de resección oncológica para el carcinoma colorectal (márgenes distal, proximal y radial así como una linfadenctomia de al menos 12 ganglios) incluso en cirugía urgente, la Guía Americana de la Sociedad de Cirujanos colorectales define los siguientes objetivos para el tratamiento urgente de esta patología: evitar el impacto negativo inmediato de la complicación, conseguir el mejor control del tumor y asegurar que el tiempo de recuperación permita el inicio del tratamiento adyuvante más adecuado. (1)(2)

\section{Obstrucción maligna de colon}

La causa más frecuente de obstrucción de colon en el adulto es la neoplasia. El carcinoma colorectal puede debutar como obstrucción en un 7-29\%, siendo la causa más frecuente de intervención urgente en estos casos ya que dejada a su evolución, la distensión progresiva del intestino progresa desde dolor abdominal con náuseas y vómitos hacia la isquemia intestinal, perforación, sepsis y posterior fallecimiento del paciente.(3)

La cirugía urgente debe realizarse respetando los principios oncológicos aunque no se disponga de un diagnostico de certeza prequirúrgico, y la intención debe ser curativa si no hay evidencia de enfermedad a distancia.(1)

La Tomografía Computarizada (TC) es la prueba radiológica de elección para localizar la lesión y la morfología de la obstrucción. Puede asociarse enema con contraste hidrosoluble, aunque no es estrictamente necesario.(4) 
Tanto el ciego como el colon derecho tienen un diámetro mayor, por lo que la obstrucción a dicho nivel es menos común y se relaciona con tumores de mayor tamaño en contexto de enfermedad avanzada, por lo que se observa una menor supervivencia libre de enfermedad en las obstrucciones por lesiones proximales en comparación con los tumores distales independientemente de las complicaciones perioperatorias o de la afectación ganglionar.(1)

\subsection{Tratamiento quirúrgico}

La actitud quirúrgica en una obstrucción neoplásica de colon ascendente suele ser una hemicolectomia derecha con ligadura de la arteria ileocólica y la rama derecha de la cólica media, con anastomosis ileotransversa. Cuando la obstrucción se localiza en el colon transverso, la resección oncológica incluye la ligadura de la arteria cólica media, con el consecuente compromiso vascular del ángulo esplénico, por lo que se realiza una hemicolectomia derecha ampliada con anastomosis ileocólica a nivel del colon descendente. Si las condiciones del paciente orientan hacia un alto riesgo de fuga anastomótica, el procedimiento más seguro es la resección del tumor con confección de ileostomia terminal, o incluso proteger la anastomosis ileocólica con ileostomia derivativa proximal.

El colon descendente y sigma son localizaciones más frecuentes de la obstrucción por neoplasia tanto por su menor diámetro como por la mayor consistencia de las heces que contiene, en comparación con las lesiones más proximales. Existen más opciones terapéuticas, aunque cada caso debe individualizarse en función del estado del paciente, experiencia del cirujano y los recursos disponibles en cada centro, no obstante existen controversias en cuanto al tratamiento más óptimo en el manejo urgente de la obstrucción distal de colon por neoplasia.(1)(5)

\subsubsection{Colostomía derivativa}

Se plantea con la intención de conseguir una resección definitiva del tumor en un segundo tiempo, con reconstrucción del tránsito incluso en una tercera intervención. En general, no se recomienda realizar una ileostomía derivativa ya que la presencia de una válvula ileocecal competente puede impedir el alivio de la obstrucción distal.

La ventaja de esta opción es que minimiza tanto el tiempo como la agresión quirúrgica en un contexto en el que paciente tiene unas condiciones tanto fisiológicas como a nivel local en la pared del colon subóptimas. También reduce 
el riesgo de contaminación y permite completar el estudio previo a decidir un tratamiento definitivo.

No obstante, la colostomía derivativa también está asociada con un alto porcentaje de complicaciones tales como el prolapso, hernia y deshidratación.

Esta intervención es segura y está indicada en caso de pacientes demasiado frágiles como para afrontar una resección intestinal, y también cuando la neoplasia es localmente avanzada con afectación de órganos adyacentes limitando así la resección oncológica adecuada en el contexto urgente.

\subsubsection{Intervención de Hartmann}

Consiste en la resección del tumor primario con la confección de una colostomía terminal, con el cierre del colon/recto distal evitando así el riesgo potencial de fuga de anastomosis, siendo ésta la intervención más frecuentemente realizada de urgencias en la obstrucción de colon distal por cáncer.

La mayor desventaja es que el estoma no está exento de complicaciones que afectan a la calidad de vida del paciente. El índice de reconstrucción es del $20 \%$ por motivos tales como enfermedad avanzada, complicaciones del tratamiento y situación basal del paciente, también se asocia con una alta morbimortalidad.(5)

\subsubsection{Resección primaria y anastomosis}

Por muchos años, la resección primaria y anastomosis en un solo tiempo quirúrgico se ha considerado demasiado arriesgado en el contexto urgente. Los motivos se justifican por una situación hemodinámica y fisiológica comprometida del paciente, mayores tiempo y agresión quirúrgica, con la dificultad añadida de manipular y movilizar un colon distendido con el riesgo de contaminación.

Los pacientes suelen estar desnutridos por la historia natural de la enfermedad, incrementando así el riesgo de complicaciones postquirúrgicas sobre todo si el intestino utilizado en la anastomosis se encuentra dilatado e isquémico, comprometiendo así las posibilidades del paciente de beneficiarse de terapia adyuvante si fuera preciso.

No obstante, la evidencia científica apoya la indicación de esta estrategia en pacientes cuidadosamente seleccionados ya que se relaciona con una menor estancia hospitalaria y evita cirugías posteriores para la reconstrucción del tránsito intestinal. Revisiones no aleatorizadas retrospectivas indican un índice de fuga anastomotica en cirugía de urgencias de entre 2,2 y $12 \%$, siendo en la cirugía electiva de entre 1,9 y $8 \%$.

La correcta selección de pacientes candidatos es la clave para el éxito de esta estrategia quirúrgica. Existen factores que se relacionan con un peor pronóstico que incluyen edad mayor a 70 años, ASA III-IV, fallo renal 
prequirúrgico, cirugía en las 24 horas tras presentación de la clínica obstructiva, y estadio avanzado de la neoplasia. Todos estos datos avocan por una intervención de Hartmann, o anastomosis con protección proximal con estoma.(1)(5)

\subsubsection{Colectomía total}

La colectomia total con anastomosis ileorectal es otra opción terapéutica en casos seleccionados en los que el colon proximal se encuentra isquémico o incluso perforado especialmente a nivel de ciego, o en casos de tumores sincrónicos o síndromes de carcinoma colorectal hereditario. Siendo estas las dos únicas indicaciones recomendadas.

\subsubsection{Endoprótesis autoexpandibles}

Representan una modalidad no quirúrgica para resolver la obstrucción maligna de colon distal. Inicialmente utilizadas en los años 90 para paliación en tumores no resecables o en pacientes no operables.

También se emplean como puente a cirugía electiva posiblemente laparoscópica.

Es una alternativa atractiva para la cirugía urgente ya que permite estabilizar al paciente y corregir su medio interno, optimizar sus comorbilidades y estado nutricional, así como completar el estudio de extensión.

Estudios recientes apoyan que al ser un puente a la cirugía, también reducen la morbilidad, mortalidad, y la necesidad de estoma en comparación con cirugía sola.(6)

No toda la evidencia apoya estas ventajas. En un estudio observacional que compara cirugía respecto al uso de stent como puente a la cirugía, no se aprecian diferencias perioperatorias en cuanto a mortalidad ni tampoco en cuanto a las tasas de anastomosis primaria ni confección de estoma respecto a cirugía sola, a pesar de que el éxito técnico en cuanto a su colocación fue del 91\% (13\% microperforación). (7)

En una revisión sistemática y metaanálisis, la tasa de éxito en cuanto a alivio de la obstrucción con endoprótesis fue solo del 52,5\%, en comparación del $99 \%$ alcanzado con la cirugía, con una morbilidad y mortalidad similares en los dos grupos, y solo se realizó anastomosis en el grupo con endoprótesis en el $64,9 \%(55 \%$ en el grupo de la cirugía), no siendo estadísticamente significativo.(8)

\section{Endoprótesis en la obstrucción maligna de colon}

Muchos pacientes con obstrucción en colon secundaria a una neoplasia se diagnostican en un estadio avanzado, son ancianos, y con deterioro clínico 
asociado a lo que se suma el riesgo de una intervención quirúrgica urgente en un colon que además no se encuentra en condiciones óptimas y sin preparación, por lo que en este contexto se plantea la necesidad de una alternativa a la cirugía de urgencias.

Tradicionalmente, el tratamiento estándar consistía en cirugías en uno o más tiempos, con morbilidad y mortalidad asociadas no despreciables, del $60 \% \mathrm{y}$ $10 \%$ respectivamente, $\mathrm{y}$ en el caso de creación de estoma, $40-60 \%$ no llegan a ser candidatos a una cirugía reconstructiva del tránsito debido al alto riesgo de la misma o por lo avanzado de su enfermedad neoplásica, además teniendo en cuenta que el estoma en sí causa un gran efecto adverso en la calidad de vida.(1)

En el caso de que se opte por una cirugía en un solo tiempo (resección y anastomosis), la intervención resulta más compleja y técnicamente difícil.

Por todos estos motivos, en general, los resultados obtenidos con la cirugía de urgencias son peores que en el contexto electivo.

\subsection{Indicaciones}

Desde que en 1991 se publicara por primera vez el uso de endoprótesis autoexpandibles por Dohomo et al, el esfuerzo de los trabajos posteriores no ha conseguido llegar a un consenso sobre la idoneidad de las indicaciones ni su generalización debido al resultado dispar de las publicaciones científicas hasta la fecha.(3)

Las dos principales indicaciones para el tratamiento de la obstrucción maligna de colon son la descompresión de la luz intestinal previo a cirugía programada (puente a la cirugía), y paliación en caso de tumores no candidatos a intervención curativa.

Las indicaciones para la colocación de endoprótesis incluyen:

Intención paliativa

Más del $19 \%$ de los pacientes con obstrucción maligna de colon tienen metástasis a distancia al diagnóstico, no siendo candidatos a cirugía curativa hasta en dos tercios de los casos. (9)

En estos pacientes con enfermedad avanzada en los que tampoco es posible una resección paliativa, puede aportar la restauración del tránsito y evitar la necesidad de estoma, con un éxito del $90 \%$ a corto plazo. La estancia hospitalaria tras el procedimiento suele ser de dos días, permitiendo el inicio precoz de la Quimioterapia (QT) paliativa.(10)

Los estudios randomizados que comparan los resultados en comparación con realización de colostomía, aportan unos resultados que demuestran la utilidad de la colocación de endoprótesis, asociándose a un precoz inicio de ingesta oral, restauración más rápida del tránsito, menor coste económico global, mejor calidad de vida y control de síntomas que con estoma. Las complicaciones a largo 
plazo incluyen perforación intestinal (10\%), migración $(9 \%)$ y reobstrucción $(18 \%) .(11)(12)$

En el consenso llevado a cabo por las sociedades de cirugía de urgencias y peritoneal en 2010 concluye que en los centros con capacidad para realizar esta técnica, los stent deberían tener preferencia respecto a la intervención con colostomía en estos casos con un grado de recomendación $2 \mathrm{~B}$, coincidiendo con la guía de la Sociedad Europea de Endoscopia.(5)

Puente a la cirugía programada

Se plantea con el objetivo de evitar la cirugía de urgencias para conseguir la optimización del paciente con descompresión intestinal y completar estudio de extensión, incluyendo la colonoscopia.

En la mayoría de las series, el tiempo medio entre la colocación del stent y la cirugía es de 7-10 días.(13). Existe poca evidencia sobre el intervalo más óptimo para realizar la cirugía programada. En teoría, más tiempo (más de una semana) permite una mejor recuperación con optimización nutricional, pero incrementa el riesgo de complicaciones relacionadas con la endoprótesis, especialmente relacionado con infiltración tumoral y reacción fibrótica. Parece que 7-10 días permite un balance adecuado entre la optimización del paciente, riesgo potencial de complicaciones del stent e impacto oncológico.(12)

El beneficio de este procedimiento es permitir la resección y anastomosis en un solo tiempo quirúrgico y evitar la confección de estomas. Además, la descompresión y optimización del paciente puede permitir una aproximación quirúrgica menos agresiva (laparoscópica). La mortalidad asociada a la colocación de stent es similar a la de la cirugía de urgencias.

La primera publicación respecto a esta indicación fue en 1994, en la que propone que el uso de stent a nivel colorectal es útil para permitir cirugía electiva posterior.(14)

Desde entonces, los estudios publicados aportan resultados contradictorios que pueden resultar confusos, aun siendo aleatorizados, incluso algunos suspendidos prematuramente (tanto por los malos resultados de esta técnica como de la intervención quirúrgica urgente)(11) (15)y otros en los que no se evidencia de la ventaja del uso de stents.(16) Sin embargo, en otros se reportan éxitos entre 85-95\%(17)(18)

Quizás pueda explicarse por la disparidad de la experiencia de los investigadores, o la habilidad de los endoscopistas pueden influir en los resultados.

En una revisión de Cochrane, que incluye 5 estudios randomizados, calculó una tasa de éxito de la endoprótesis en un $86,02 \%$, con complicaciones asociadas en un $39,22 \%$, similar al $45,71 \%$ asociado a la cirugía de urgencias. Las ventajas son una estancia hospitalaria más corta, siendo el procedimiento más 
rápido y con menos sangrado asociado. Concluye que los resultados clínicos dependen del éxito de la técnica, que puede variar dependiendo de la experiencia y habilidad de los que realizan el procedimiento.(19)

Otro factor a tener en cuenta es el efecto traumático de la endoprótesis sobre la neoplasia, con la posibilidad de perforación subclínica con el riesgo de diseminación tumoral. No obstante la obstrucción en sí empeora el pronóstico del paciente.

En un estudio retrospectivo en cuanto al uso de endoprótesis como puente a la cirugía se evidenció un descenso significativo de la supervivencia a 5 años en el grupo del stent respecto con cirugía sola ( 25 vs $62 \%$ respectivamente), la mortalidad debida a cáncer también fue superior en el grupo con stent (48\% vs $21 \%$ en el grupo de cirugía sola). Tampoco hubo beneficio significativo en el grupo de cirugía sola en cuanto a supervivencia libre de enfermedad, recurrencia, y tiempo libre de enfermedad. En el estudio multivariante, la colocación de stent fue el único factor modificable que afectaba a un peor resultado en ese brazo del estudio.(20)

En el aspecto económico, la endoprótesis seguida de cirugía electiva es significativamente más efectiva y eficiente que la cirugía de urgencias.

En el consenso de las sociedades de cirugía de Urgencias y peritoneal se apoya la idea de que la endoprótesis debería usarse como puente la cirugía programada en los centros de referencia con experiencia y en pacientes seleccionados ya que se asocia con una menor mortalidad, menor estancia hospitalaria y menor tasa de colostomía asociada, con un grado de recomendación 1B. (5). Esta recomendación coincide con la de la Sociedad Europea de Endoscopia, que tampoco considera que la endoprótesis deba ser una procedimiento estándar para aquellos pacientes potencialmente curables, apuntando su idoneidad para aquellos con un mayor riesgo quirúrgico (ASA igual o superior a III, y edad mayor a 70 años).(12)

En el manejo de pacientes con tumores pélvicos extracolónicos por compresión extrínseca.

Las neoplasias extracolónicas pueden causar obstrucción de su luz. Los tumores primarios más frecuentes suelen ser ginecológicos, pancreáticos, vesicales, prostáticos, o por invasión de la pared del colon.

Suelen causar estenosis en más de un segmento intestinal, y puede asociar carcinomatosis, que junto con el antecedente de radioterapia en algunos casos, son factores que condicionar el fracaso del stent.

En general, en manos de endoscopistas expertos, las tasas de éxito superan a la de complicaciones, no obstante se precisan de más estudios antes de considerar a la endoporótesis como estándar en el tratamiento de la obstrucción maligna de colon. En el caso de enfermedad metastásica o esperanza de vida 
corta, los stent pueden prevenir las complicaciones quirúrgicas al evitar la intervención, y permitir un rápido inicio o continuar con QT sistémica.(12)(18)

\subsection{Contraindicaciones}

a) En caso de pacientes con peritonitis e inestabilidad hemodinámica en cuya situación se recomienda cirugía urgente, y en aquellos en los que se sospeche isquemia y/o perforación.

b) También está contraindicado en caso de absceso intraabdominal.

c) Relativo en situaciones de persistencia de coagulopatía a pesar de tratamiento.

d) Lesiones rectales distales ( $5 \mathrm{~cm}$ de margen anal): el stent en estos casos puede causar síntomas como dolor, tenesmo y sangrado.

e) Carcinomatosis peritoneal diseminada, con estenosis multifocales a nivel intestinal(9)(18)

\subsection{Complicaciones}

La colocación de endoprótesis es un procedimiento de bajo riesgo con una mortalidad inferior al $1 \%$.

Pueden clasificarse entre tempranas o tardías, si ocurren antes o después de los 30 días del procedimiento. La perforación, el sangrado y el mal posicionamiento suelen ser tempranas. La migración, reobstrucción, tenesmo y perforación se relacionan con una aparición más tardía.

En una revisión sistemática (Sebastian y cols.), las endoprotesis autoexpandibles están asociadas con un índice de complicaciones cercano al 25\%. La complicación más frecuente en un total de 1198 pacientes fue la migración del stent (12\%), recurrencia de la obstrucción (7\%), y perforación (4\%).(18)

\section{Perforación}

Es la complicación más grave en relación con la endoprótesis, con un riesgo total del 5\%. Puede ser inmediata o diferida, y suele ser más frecuente en el colon distal donde la angulación y redundancia del mismo pueden dificultar el acoplamiento de la endoprótesis. Además, en el colon izquierdo, los pliegues de la mucosa pueden colgar en los dos extremos del stent y causar excesiva tensión en los puntos de apoyo con la consecuente necrosis.(12)

La perforación relacionada con el procedimiento supone el 15-20\% de los casos, por la manipulación de la guía y dilatación. La sobredistensión neumática de un colon ya distendido puede resultar en una perforación en asa cerrada, sobre 
todo a nivel tumoral por la tensión circunferencial de la luz, o por su apoyo en una mucosa friable en el margen de la lesión.

Suele requerir de cirugía urgente, además, la contaminación peritoneal añade un estrés fisiológico al paciente que puede limitar las opciones quirúrgicas.

Algunos autores incluso argumentan que tras una colocación sin incidencias, el trauma local producido por el stent puede originar diseminación tumoral con el empeoramiento oncológico subyacente.(21)(22)

Se ha evidenciado una alta incidencia en los pacientes con QT, corticoides y Radioterapia (RT) que incluso triplica el riesgo de perforación, que es más precoz con bevacizumab. Por lo que se no se recomienda la colocación de endopróteis en pacientes que van a recibir tratamiento con agentes antiangiogénicos.(18)

\section{Migración}

Suele ocurrir durante la primera semana aunque puede suceder más tarde, a consecuencia de que el stent sea estrecho, pequeño o corto, que la lesión no sea obstructiva (compresión extrínseca), por impactación fecal, o porque el tumor reduzca en tamaño tras RT o QT.

Las tasas de migración para las endoprótesis no cubiertas son del 3\%$12 \%$, aumentado al $30 \%-50 \%$ para las cubiertas.

Puede ser asintomática o recurrir la obstrucción, otros síntomas son el sangrado o tenesmo si se localiza en el anorecto.

En las migraciones distales, su retirada no es técnicamente difícil, incluso puede ser manual sin precisar endoscopio.

\section{Oclusión}

Suele deberse a crecimiento tumoral a nivel intraluminal, y se considera un factor importante de los resultados a largo plazo del stent. La probabilidad de que ocurra aumenta con el tiempo tras su colocación por la evolución natural de la neoplasia y tendencia a la invasión local. Es más frecuente en el contexto paliativo.

Puede resolverse con la introducción de una endoprótesis adicional.

Los factores que se asocian con una evolución más favorable son el uso de una endoprótesis inferior a $10 \mathrm{~cm}$ en longitud, y en los pacientes con obstrucción distal.

Sangrado

Lo habitual es que sea mínimo en relación con el procedimiento y suele deberse a la friabilidad del tumor, por lesión del mismo o irritación de la mucosa. Un sangrado más tardío se relaciona a ulceración o erosión de la mucosa del colon.(23)

\section{Dolor}


El dolor abdominal moderado y transitorio es frecuente y puede durar incluso hasta cinco días tras el procedimiento ya que la expansión de la endoprótesis ocurre en las primeras 48-72 horas. En general no requiere de tratamiento específico salvo analgesia. (18)

\subsection{Tipos y composición de las endoprótesis}

Los materiales disponibles para las prótesis colónicas son el acero inoxidable, Elgiloy y nitinol, siendo este último el más utilizado. El nitinol es una aleación de níquel y titanio que tiene memoria en su forma, una gran elasticidad y es compatible con la resonancia magnética. La estructura consiste en una malla cilíndrica que una vez desplegada, se expande en las 24-72 horas siguientes incorporándose así en el tumor y tejido circundante por presión, este efecto es mayor en las prótesis no cubiertas ya que los huecos del entramado de su estructura están libres, reduciendo así el riesgo de migración. Los stent cubiertos tienen esos huecos cerrados (poliuretano, polietileno o silicona), impidiendo así el crecimiento intratumoral, tienen la ventaja de impedir la estenosis, pero una mayor tendencia a la migración. La elección de uno $\mathrm{u}$ otro tipo depende del propósito buscado. Así, cuando la indicación es como puente a la cirugía se prefieren los no cubiertos ya que se prioriza la permeabilidad y fijación, puesto que la cirugía se programa en corto plazo. Para la paliación se opta por stent cubiertos ya que van permanecer por largos periodos de tiempo, impidiendo así el crecimiento intraluminal y la reobstrucción a más largo plazo. Existen endoprótesis biodegradables, desarrolladas originalmente para tratar estenosis benignas que están hechas de polímeros sintéticos, principalmente poliglicólidos, polidioxanona y poliláctidos. La vida media de degradación in vitro varía dependiendo del diámetro de la fibra, la estructura de la malla, el tipo de tejido donde se colocó, el tipo de material biodegradable utilizado, el $\mathrm{pH}$ y la temperatura.(9)(18)

\subsection{Preparación y procedimiento}

Antes de proceder a la colocación de la endoprótesis, es necesaria una evaluación radiológica mediante $\mathrm{TC}$ ya que esta prueba cuenta con una sensibilidad de $96 \%$ y especificidad de $93 \%$ para localizar la lesión obstructiva además de aportar información sobre la invasión local y a distancia de la misma, la especificidad puede aumentar hasta el $100 \%$ si se asocia enema de contraste hidrosoluble, aunque es menos usado en la práctica habitual.(12) 
Preparación intestinal: depende de la localización y grado de obstrucción. En pacientes con obstrucción parcial a nivel distal en el colon, suele ser suficiente con dos enemas de limpieza Si la obstrucción es parcial en una lesión más proximal, puede intentarse una preparación mecánica oral que se suspende en caso de sintomatología como dolor o distensión abdominal así como náuseas o vómitos. En pacientes con obstrucción completa, la preparación oral no es tolerada y puede precipitar la perforación intestinal. Los enemas sí se recomiendan para evacuar las heces a nivel distal.

Antibióticos: no es necesaria la profilaxis antibiótica, no obstante se recomienda en casos de obstrucción completa en aquellos pacientes con una marcada dilatación de colon ya que la insuflación durante el procedimiento puede provocar microperforación y bacteriemia.

Los métodos de colocación incluyen fluoroscopia, colonoscopia, y combinación de ambas. En el recto y colon distal se puede elegir uno de los 2 métodos, pero para lesiones más proximales se recomienda un abordaje mixto. La colonoscopia puede ayudar al abordaje y paso de la guía, y la fluoroscopia es útil para documentar la extensión de la obstrucción y para el diagnóstico de perforación.

Durante la endoscopia, la insuflación debe ser la mínima necesaria para reducir el riesgo de perforación debido al fenómeno de obstrucción en asa cerrada entre la lesión y la válvula ileocecal.

Se introduce una guía a través de la lesión con la asistencia de fluoroscopia, seguido de la endoprótesis, una vez expandida se comprueba el éxito del procedimiento con insuflación de aire y liquido a través de su trayecto, incluso puede avanzar el endoscopio para visualizar el colon proximal. Aunque es un procedimiento factible para todo el colon, está más desarrollado y estudiado para lesiones izquierdas.(18)

\section{Bibliografía}

1. BAER C, MENON R, BASTAWROUS S « Emergency presentations of colorectal Cancer » Surgical Clinics of North America, vol 97, p.529-545. 2017

2. TEXEIRA F, AKAISHI EH, USHINOHAMA AZ « Can we respect the principles of oncologic resection in an emergency surgery to treat colon cancer? » vol 10. Pp 5-15. 2015 
3. CHEUNG HY, CHUNG CC, TSANG WW «Endolaparoscopic approach vs conventional open surgery in the treatment of obstructing left-sided colon cancer: a randomized controlled trial», Archives of Surgery, vol 144, pp.1127. 2009

4. FRAGO R, RAMIERZ E, MILLAN $M$ « Current management of acute malignant largebowel obstruction: a systematic review » American Journal of Surgery, vol 207, PP.127-38.2014

5. ANSALONI L, ANDERSSON RE, BAZZOLI $\mathrm{F}$ « Guidelines in the management of obstructing cáncer of the left colon: consensus conference of the worlde society of emergency surgery (WSES) and peritoneum and surgery (PnS) society » World Journal of Emergency Surgery, vol 5, pp.29-39. 2010

6. KWAK MS, KIM WS, LEE JM « Does stenting as a bridge to surgery in leftsided colorectal cáncer obstruction really worsen oncological outcomer?» Diseases of Colon and Rectum, vol 59, pp. 725-32. 2016

7. KVANAGH DO, NOLAN B, JUDGE C. «A comparative study of short- and medium-term outcomes comparing emergent surgery and stenting as a bridge to surgery in patients with acute malignant colonic obstruction » Diseases of Colon and Rectum, vol 56, pp. 433.40. 2013

8. CIROCCHI R, FARINELLA E, TRASTULLI S. "Safety and efficacy of endoscopic colonic stenting as a bridge to surgery in the management of intestinal obstruction due to left colon and rectal cancer: a systematic review and meta-analysis». Surgical Oncology, vol 22, $n^{\circ} 1$, pp. 14-21. 2013

9. YOUNG CJ, DE-LOYDE KJ, YOUNG JM «Improving Quality of Life for People with Incurable Large-Bowel Obstruction: Randomized Control Trial of Colonic Stent Insertion » Diseases of colon and rectum, vol 58, p.838. 2015

10. ABBOTT, S, EGLINTON TW, MA Y. "Predictors of outcome in palliative colonic stent placement for malignant obstruction», British Journal of Surgery, pp.34-42. 2014

11. VAN HOOFT JE, BEMELMAN WA, OLDENBURG B «Colonic stenting versus emergency surgery for acute left-sided malignant colonic obstruction: a multicentre randomised trial » Lancet Oncology, vol 12, pp. 344-352. 2011 
12. VAN HALSEMA EE, VAN HOOFT JE, SMALL AJ «Perforation in colorectal stenting: a meta-analysis and a search for risk factors " Gastrointestinal Endoscopy, vol 79, pp. 970-75. 2014

13. WATT AM, FARAGHER IG, GRIFFIN TT « Self-expanding metallic stents for relieving malignant colorectal obstruction: a systematic review», Annals of Surgery, pp.246-24. 2007

14. CHEUNG DY, LEE YK, YNAG CH «Status and literatura review of SelfExpandable Metallic Stents for Malingnant Colorectal Obstruction »Clinical Endoscopy, vol 47, pp. 65-73. 2014

15. ALCANTARA M, SERRA.ARACIL X, FALCO J «Prospective, controlled, randomized study of intraoperative colonic lavage versus stent placement in obstructive left-sided colonic cancer " World Journal of Surgery, vol 35, pp. 1904-1910. 2011

16. PIRLET IA, SLIM K, KWIATKOWSKI F « Emergency preoperative stenting versus surgery for acute left-sided malignant colonic obstruction: a multicenter randomized controlled trial » Surgical Endoscopy, vol 25, pp. 1814-1821. 2011

17. SEBASTIAN S, JOHNSTON S, GREOGHEGAN T «Pooled analysis of the efficacy and safety of self-expanding metal stenting in malignant colorectal obstruction» American Journal of Gastroenterology, vol 99, pp. 2051-64. 2004

18. BARON TH «Colonic stenting: technique, technology, and outcomes for malignant and benign disease » Gastrointestinal Endoscopy Clinics of North America, vol 15, pp. 757-771. 2005

19. SAGAR J « Colorectal stents for the management of malignant colonic obstructions » Cochrane Database Syst Rev 2011

20. SABBAGH C, BROWET F, DIOUF M « Is stenting as "a bridge to surgery" an oncologically safe strategy for the management of acute, left-sided, malignant, colonic obstruction? A comparative study with a propensity score analysis » Annals of Surgery, vol 258, pp. 107-15. 2013

21. MARUTHACHALAM K, LASH GE, SHETON BK « Tumour cell dissemination following endoscopic stent insertion » British Journal of Surgery, vol 94, pp.1151-4. 2007 
22. KIM SJ, KIM HW, PARK SB «Colonic perforation either during or after stent insertion as a bridge to surgery for malignant colorectal obstruction increases the risk of peritoneal seeding » Surgical Endoscopy, vol 29, pp. 3499-3505. 2015

23. KAPLAN J, STRONGIN A, ADLER DG « Enteral stents for the management of malignant colorectal obstruction » World Journal of Gastroenterology, vol 20, pp. 132-39. 2014 